\title{
The Main Determinants for Suicidal Ideation in a Romanian Cohort of Multiple Sclerosis Patients
}

\author{
Andreea Romaniuc $\mathbb{D}^{1,}{ }^{1,2}$ Rodica Bălaşa, ${ }^{1,3}$ Nicoleta Ştirbu $\mathbb{D}^{4},{ }^{4}$ Smaranda Maier, ${ }^{1,3}$ \\ Sebastian Andone, ${ }^{1}$ Zoltan Bajko, ${ }^{1,3}$ Laura Bărcuţean $\mathbb{D}^{\mathbb{1}},{ }^{1,3}$ Septimiu Voidăzan $\mathbb{D}^{5}{ }^{5}$ \\ and Anca Moțăţăianu $\mathbb{D}^{1,3}$ \\ ${ }^{1}$ Neurology Clinic I, Emergency Clinical County Hospital, Targu Mures, Romania \\ ${ }^{2}$ Doctoral School (I.O.S.U.D.) of the University of Medicine, Pharmacy, Science and Technology of Târgu Mureș, Romania \\ ${ }^{3}$ Department of Neurology, University of Medicine, Pharmacy, Science and Technology of Targu Mures, Romania \\ ${ }^{4}$ Municipal Hospital, Sighisoara, Romania \\ ${ }^{5}$ Department of Epidemiology, University of Medicine, Pharmacy, Science and Technology of Targu Mures, Romania
}

Correspondence should be addressed to Nicoleta Ştirbu; degetar_nicoleta@yahoo.com

Received 30 September 2019; Revised 26 November 2019; Accepted 6 December 2019; Published 21 January 2020

Academic Editor: Jesus Pastor

Copyright (c) 2020 Andreea Romaniuc et al. This is an open access article distributed under the Creative Commons Attribution License, which permits unrestricted use, distribution, and reproduction in any medium, provided the original work is properly cited.

\begin{abstract}
Objective. To determine the prevalence of suicidal concerns (SC) in a large multiple sclerosis (MS) patient group and to assess the major determinants that are implicated in their occurrence. Methods. A total of 349 patients were included in the study. They completed a survey about their demographic characteristics, psycho-socio-economic data, and disease-related information. Their disability level was assessed using the Expanded Disability Status Scale (EDSS) based on the neurological examination performed by the same doctor for every patient and the SC were documented with the Beck Depression Inventory-II questionnaire. Results. The study included 112 men and 237 women, with a mean age around 42 years old. Suicidal thoughts were more frequent in men, while suicidal intentions in women. Positive correlations were found between SC and depression, EDSS, total number of relapses, disease duration, and level of education. From the EDSS functional scores, only the pyramidal score and the cerebellar score presented a significant correlation with SC. None of the patients with clinically isolated syndrome had SC. The type of disease-modifying therapy, marital and occupational status, and the presence of children did not influence the presence of SC. Conclusions. The prevalence of SC is higher in patients with MS compared to the general population. Their occurrence is mostly influenced by the disease itself (duration, relapses, acquired disability) and also by depression and lack of education.
\end{abstract}

\section{Introduction}

Multiple sclerosis (MS) is known as one of the most common disabling conditions among young people. Although the most striking symptoms appear to be the ones that cause the physical disability (such as visual loss, motor deficits, sensitive, and cerebellar symptoms), the psychological and psychiatric manifestations of the disease (such as depression, behavioral changes), that can sometimes act as "silent killers" leading to suicidal ideation, have come to the attention of researchers over the last several years. Even though
Charcot was the first (in the nineteenth century) who described MS's psychiatric manifestations in his lectures at Salpetriere Hospital, these aspects were only more recently debated, playing an important part of the quality of life in MS patients. These symptoms can be easily missed at a routine follow-up visit, especially if the patient has good coping mechanisms $[1,2]$.

The most frequently reported psychiatric symptoms are depression, dysphoria, agitation, anxiety, and irritability $[3,4]$. In the development of depression in the MS patients, a crucial factor, with respect to the psychological 
aspects of the disease, is the strain of being diagnosed with an incurable, progressive, and unpredictable condition [5]. Although MS is defined as a progressive, neurodegenerative disorder, according to Rolak, the life expectancy is reduced only by a few months and after 15 years from the onset only about $20 \%$ of patients will be restricted to their beds [6]. However, not every patient is willing to accept these statistics. Depression in MS is not only a frequent feature of the clinical picture but is also among the most important determinants for suicidal intent and completion $[7,8]$.

Suicide has always been a tragic, emotional, debatable, and controversial subject from many points of view. Suicide is defined as the act of deliberately ending one's life. Suicidal behavior, which can be explained as an individual's elaborated set of strategies, ideas, and actions designed to commit suicide, is classified in an escalating order into suicidal ideation, plan, and attempt. Suicidal behavior is far more common (10-20 times) than completed suicide, but it remains officially underreported due to social, legal, and economic implications $[9,10]$.

According to World Health Organization's data from 2015, one person dies every 40 seconds by committing suicide, this being the second leading cause of death in the 15-29-year-old age group [11]. Suicide attempt is more frequent in women, unmarried people, and patients diagnosed with a psychiatric disorder, whereas suicide completion is more common in men [12]. Regarding suicide in MS patients, it was shown that the risk is twofold higher than that in the general population and is particularly increased for men receiving this diagnosis and within the first year of diagnosis [13].

The aim of the present study was to assess the prevalence and the determinants for suicidal concerns (SC) in a large MS patient group.

\section{Methods}

2.1. Patients. We performed an observational, prospective, and cross-sectional study. Three hundred forty-nine patients, who consecutively attended our Regional MS Centre over a six-month period, were included in this study. Inclusion criteria consisted of several parameters: (1) age over 18 years; (2) diagnosis of clinically isolated syndrome (CIS), relapsingremitting MS (RRMS), and/or secondary progressive MS (SPMS) according to McDonald criteria 2010 [14]; (3) undergoing treatment with interferon (IFN) beta-1a and beta- $1 \mathrm{~b}$, glatiramer acetate, and natalizumab (these were the only disease-modifying therapies available in Romania at the time of the study); and (4) signed informed consent to participate in the study. The exclusion included several parameters: (1) recurrence with corticosteroid treatment in the last month; (2) diagnosis of depression or concomitant treatment with antidepressants; (3) participated in clinical trials on experimental therapy; (4) diagnosed with other chronic diseases (neoplasia, epilepsy); and (5) surgery or hospitalization in the last month. All of the patients completed a survey about their demographic characteristics, psychosocio-economic data (marital and occupational status, presence of children, education), and disease-related information (disease and disease-modifying therapy (DMT) duration, type of DMT, MS course, number of relapses). Their disability levels were evaluated using the Expanded Disability Status Scale (EDSS), which was obtained by the same neurologist for all patients [15]. SC were documented with questions from Beck Depression Inventory-II (BDI-II), the Romanian version from 2012 [16]. The patients who reported that they had been thinking about committing suicide in the last 2 weeks, but would not do so (score 1), were considered to have suicidal thoughts (ST) and those who reported that had intended to commit suicide (score 2) or that would take life if given the chance (score 3 ) were considered to have suicidal intentions (SI). The study was conducted in accordance with the principles set out in the Helsinki Declaration. All patients signed the informed consent.

2.2. Statistical Analysis. We used the Statistical Package for Social Sciences for all statistical calculations [17]. The Shapiro-Wilk test was used to assess the normality of continuous variables. The difference between means of continuous variables was assessed using Student's $t$-test (expressed as the mean \pm standard deviation), while differences between nonparametric variables (express as median, range) were compared with the Mann-Whitney test. The KruskalWallis test was used to estimate the difference among the other variables, which is appropriate for more than two groups. Associations between categorical variables were assessed using contingency tables and chi-squared test. Correlations between quantitative variables were analyzed with Spearman's rho. All tests were interpreted against a $p=0.05$ significance threshold; statistical significance was considered for values below this threshold.

\section{Results}

The demographic-, psycho-socio-economical-, and MSrelated data are presented in Table 1.

From the entire group of MS patients, 314 (89.97\%) patients reported not thinking to take their life (score 0), 17 (4.87\%) were thinking about committing suicide but would not do so (score 1), 15 (4.29\%) intended to commit suicide (score 2 ), and $3(0.87 \%)$ would take their own life if given the chance (score 3 ).

The study included 112 men and 237 women. We observed no statistically significant differences regarding the presence of suicidal ideas and intents between the two sexes. ST were found in $3.8 \%$ of women and $7.1 \%$ of men, while SI were observed in $5.9 \%$ of women and only $2.7 \%$ in men.

Using univariate analysis and a Spearman correlation, we obtained positive correlations between SC and disease duration $(p=0.027, r=0.118)$ and the number of relapses, respectively $(p=0.045, r=0.110)$ (Figure 1 ).

The influence that education had on SC is presented in Figure 2. Most patients with a university degree (92.3\%) and $95.7 \%$ of the postgraduates had a score of 0 , while this score was found in only $69.5 \%$ of those with secondary school studies. SI were found in $30.8 \%$ of patients with secondary education and only in $1.9 \%$ of those with university education. The differences were statistically significant, $p=0.021$. 
TABLE 1: Demographic-, psycho-socio-economical-, and MS-related data.

\begin{tabular}{|c|c|c|c|}
\hline & $\begin{array}{l}\text { Without ST and SI } \\
0\end{array}$ & $\begin{array}{c}\text { With ST } \\
1\end{array}$ & $\begin{array}{c}\text { With SI } \\
2-3\end{array}$ \\
\hline \multicolumn{4}{|l|}{ Gender } \\
\hline Female & $213-67.9 \%$ & $9-52.9 \%$ & $15-83.3 \%$ \\
\hline Male & $101-32.2 \%$ & $8-47.1 \%$ & $3-16.7 \%$ \\
\hline Age (mean/SD) & $42.85 / 9.75$ & $42.59 / 8.91$ & $45.5 / 8.71$ \\
\hline \multicolumn{4}{|l|}{ Disease duration } \\
\hline Median (min-max) & $10(0-39)$ & $10(2-28)$ & $11(7-34)$ \\
\hline \multicolumn{4}{|l|}{ Type of MS } \\
\hline CIS & $10-3.2 \%$ & $0-0.0 \%$ & $0-0.0 \%$ \\
\hline RRMS & $224-71.3 \%$ & $11-64.7 \%$ & $10-55.6 \%$ \\
\hline SPMS & $80-25.5 \%$ & $6-35.3 \%$ & $8-44.4 \%$ \\
\hline \multicolumn{4}{|l|}{ Treatment duration } \\
\hline Median (min-max) & $7(0-15)$ & $9(1-14)$ & $7.5(3-13)$ \\
\hline \multicolumn{4}{|l|}{ Type of treatment } \\
\hline AVONEX & $44-14 \%$ & $3-17.6 \%$ & $0-0.0 \%$ \\
\hline BETAFERON & $121-38.5 \%$ & $7-41.2 \%$ & $9-50 \%$ \\
\hline COPAXONE & $58-18.5 \%$ & $2-11.8 \%$ & $6-33.3 \%$ \\
\hline REBIF & $78-24.8 \%$ & $2-11.8 \%$ & $3-16.7 \%$ \\
\hline TYSABRI & $13-4.1 \%$ & $3-17.6 \%$ & $0-0.0 \%$ \\
\hline \multicolumn{4}{|l|}{ EDSS } \\
\hline Median (min-max) & $2.5(0-8.5)$ & $4(1.5-8)$ & $3.25(1-7)$ \\
\hline \multicolumn{4}{|l|}{ Number of relapses } \\
\hline Median (min-max) & $4(0-24)$ & $5(1-32)$ & $5.5(1-15)$ \\
\hline \multicolumn{4}{|l|}{ Marital status } \\
\hline Married/living with partner & $210-67.1 \%$ & $9-52.9 \%$ & $14-77.8 \%$ \\
\hline Single & $68-21.7 \%$ & $5-29.4 \%$ & $3-16.7 \%$ \\
\hline Divorced & $23-7.3 \%$ & $3-17.6 \%$ & $1-5.6 \%$ \\
\hline Widow & $12-3.8 \%$ & $0-0.0 \%$ & $0-0.0 \%$ \\
\hline \multicolumn{4}{|l|}{ Educational level } \\
\hline Elementary school & $9-2.9 \%$ & $0-0.0 \%$ & $4-22.2 \%$ \\
\hline Apprentice school & $54-17.3 \%$ & $4-23.5 \%$ & $5-27.8 \%$ \\
\hline High school & $119-38 \%$ & $7-41.2 \%$ & $6-33.3 \%$ \\
\hline University & $96-30.7 \%$ & $6-35.3 \%$ & $2-11.1 \%$ \\
\hline Master or PhD & $22-7.0 \%$ & $0-0.0 \%$ & $1-5.6 \%$ \\
\hline Technical and vocational high school & $13-4.2 \%$ & $0-0.0 \%$ & $0-0.0 \%$ \\
\hline \multicolumn{4}{|l|}{ Occupational status } \\
\hline Employed & $125-39.9 \%$ & $6-35.3 \%$ & $4-22.2 \%$ \\
\hline Part-time employed & $20-6.4 \%$ & $1-5.9 \%$ & $1-5.6 \%$ \\
\hline Disability pension & $140-44.7 \%$ & $10-58.8 \%$ & $13-72.2 \%$ \\
\hline Retired & $8-2.6 \%$ & $0-0.0 \%$ & $0-0.0 \%$ \\
\hline Student & $2-0.6$ & $0-0.0 \%$ & $0-0.0 \%$ \\
\hline Unemployed & $17-5.4 \%$ & $0-0.0 \%$ & $0-0.0 \%$ \\
\hline
\end{tabular}

Occupational status did not significantly influence the presence of suicidal ideas $(p=0.998)$. No statistically significant differences regarding the presence or absence of suicidal ideas were registered among the four patient groups, that were divided according to marital status $(p=0.909)$. We believe, however, that it is important to note that of the 18 patients with SI, $77 \%$ were married, $16.6 \%$ were unmarried, and $5.5 \%$ were divorced (Figure 3 ).

Concerning the clinical form of MS, $100 \%$ of CIS patients had a score of 0 . SI were traced to $4.1 \%$ of patients with RRMS and $8.5 \%$ of those with SPMS. The difference was not statistically significant $(p=0.69)$ (Figure 4 ). 

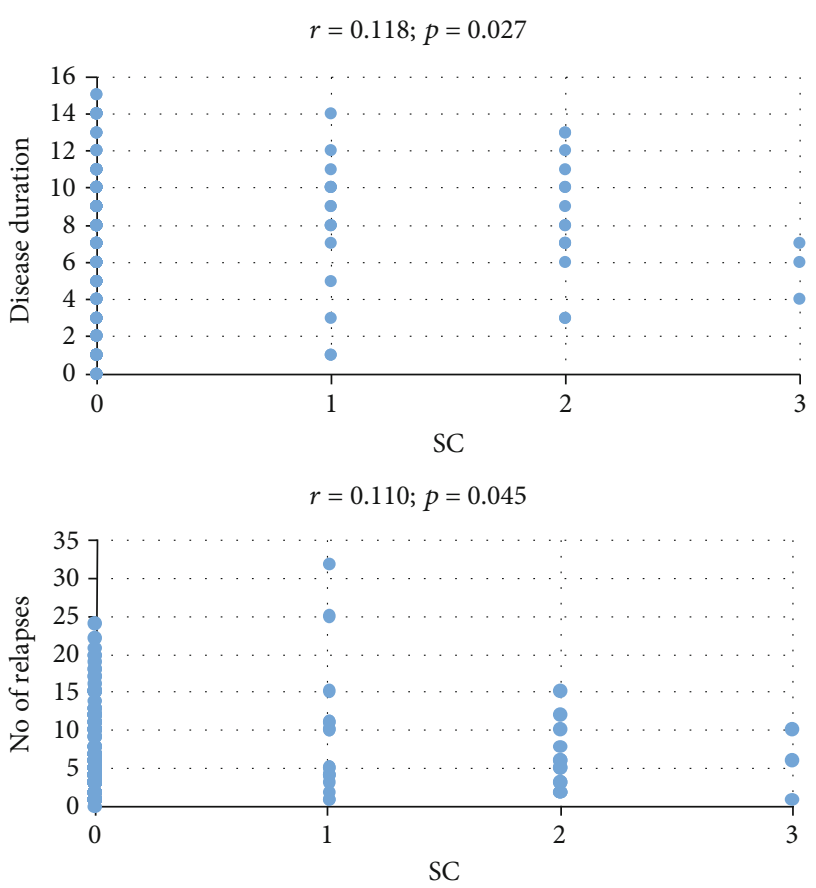

Figure 1: Correlation between SC and disease duration and between the number of relapses and SC.

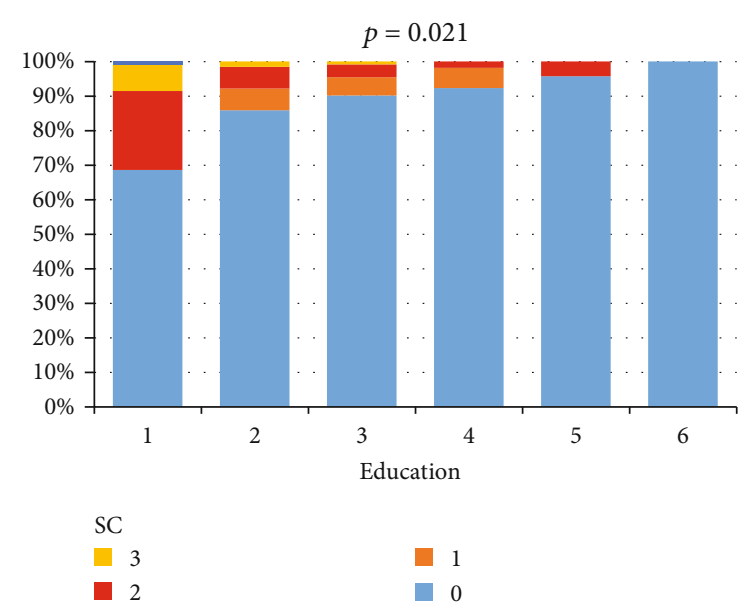

Figure 2: The relationship between SC and education level: 1-elementary school, 2-apprentice school, 3-high school, 4-university, 5-master or $\mathrm{PhD}$, and 6-technical and vocational high school.

SC presence and severity was positively correlated with the EDSS score ( $p=0.001, r=0.171)$ (Figure 5 ). Of the eight functional scores, SC only correlated with the pyramidal (PFS) and cerebellar (CFS) ones $(p=0.02, p=0.005)$. We found no SI nor ST for $92.7 \%$ of the patients with a PFS of 0 and only for $50 \%$ of those with a PFS of 5 . SI were observed in $3.6 \%$ of the patients with PFS 0 and $14 \%$ of those with PFS 4. Most patients with CFS 0 (92.4\%) had no suicidal ideas or SI, and only $66.7 \%$ of those with a score of 4 were in the same

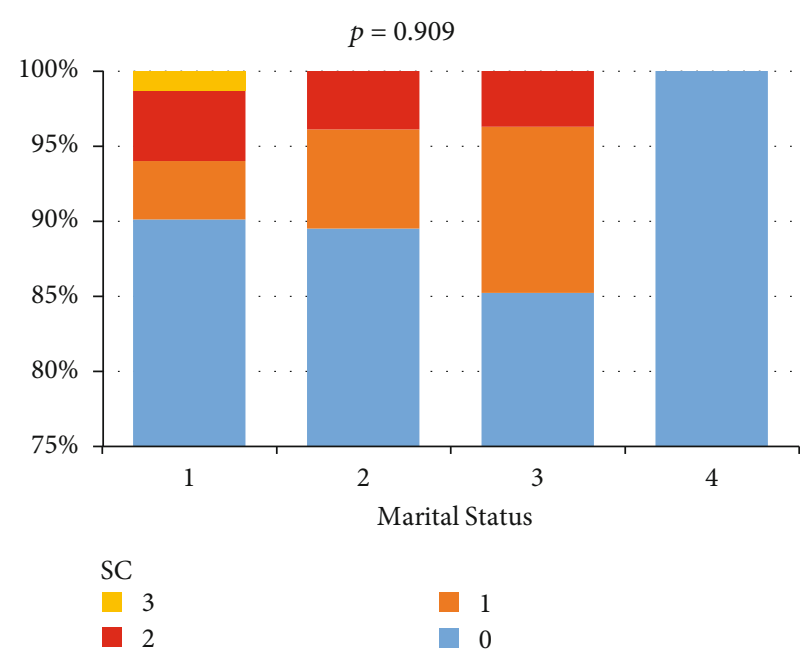

Figure 3: The relationship between SC and marital status: 1-married/living with partner, 2-single, 3-divorced, and 4-widow.

situation. SI were present in $3.5 \%$ of patients with CFS 0 and $33.3 \%$ of those with CFS 4.

The type of DMT, MS course, marital and occupational statuses, and presence of children did not influence the prevalence of SI.

SC presence and severity was strongly correlated with the total score obtained from the BDI-II questionnaire $(p=0.0001)$. The majority of patients with BDI-II score $<10$ (98.9\%) have not had SI or ST, and only $54.2 \%$ of those with BDI-II score $\geq 31$ were included in the same category. SI were observed in $0 \%$ of those without mood disorders and with mild disturbances, in $8.3 \%$ of those with borderline depression, $17.2 \%$ of those with moderate depression, and $37.5 \%$ of those with severe depression (Figure 6).

\section{Discussion}

Over the past few years, the issue of psychiatric manifestations and especially the suicide matter in MS patients had attracted the attention of many researchers.

It has been established by the epidemiological data that the suicide rate in these patients is higher than that in the general population. Most of the information was retrieved from the Northern European countries thanks to their extensive databases.

The standardized mortality ratio (SMR) was the most used method to quantify the suicide rate. SMR represents the number of deaths in people with MS in a given period divided by the number of deaths expected in the general population on the same loop of time. A value $>1.0$ shows an increased suicide rate with or without statistical significance [18].

Different time-lapse studies based on large numbered population cohorts (ranging from 1595 to 12834 people) returned significant SMR values as it follows: 2.3 (from a Swedish research) [13], 1.62, 1.83, and 2.12 from three Danish studies [19-21] and 1.7 from a Finnish study [22]. Other investigators used a different tool to estimate the 

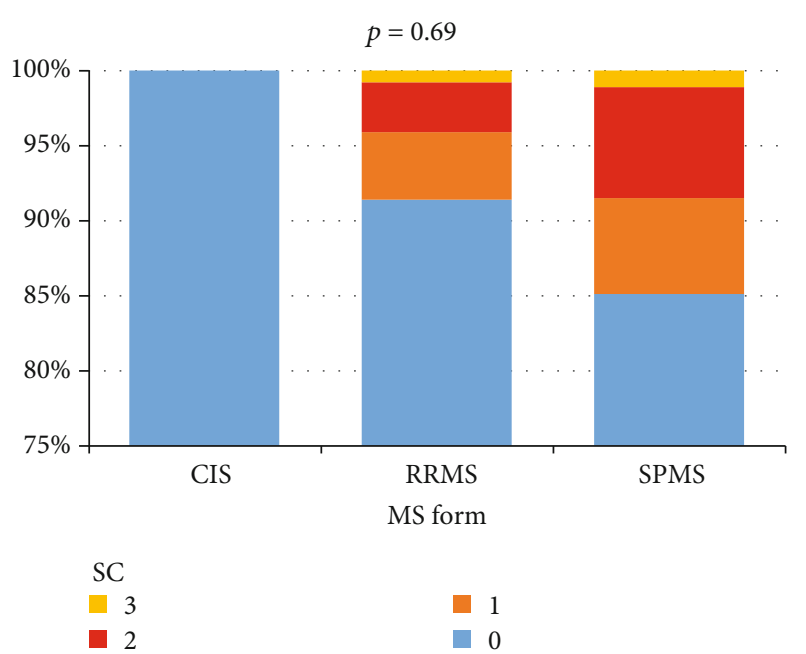

FIGURE 4: The relationship between SC and MS form.

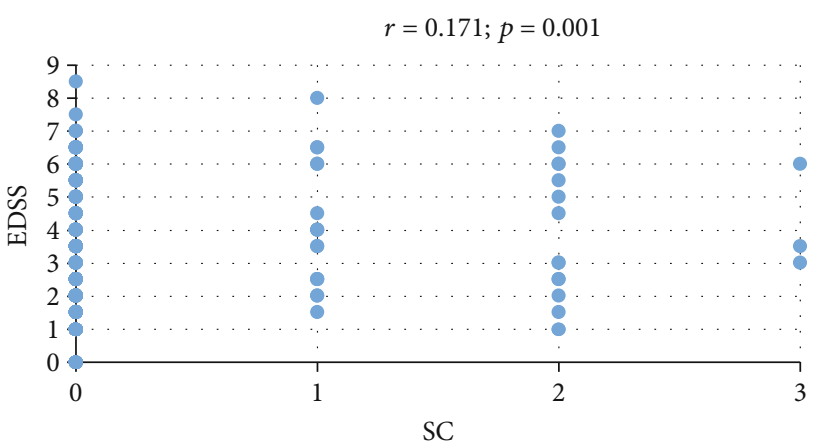

Figure 5: Correlation between the EDSS and SC.

completed suicide rate by returning a hazard value (which is an estimation from a Cox regression analysis also adjusted for demographics and timeline) of 1.87 (95\% confidence interval: $1.53-2 / 3$ ) similar to the ones described above [23].

The present study also is aimed at drawing the attention towards the psychiatric aspects of MS, quantifying the prevalence of SC and searching for their determinants in a large MS patient group.

The prevalence of suicidal behavior data presents fluctuations in the literature. Its variability could be explained by the heterogeneity of the studied groups and also by the methods used for interviewing the patients.

Three studies have applied the Patient Health Questionnaire which includes nine questions such as "Little interest or pleasure in doing things?" or "Thoughts that you would be better off dead or of hurting yourself in some way?". The subject answers each item from "not at all" to "nearly every day" adapting it to the events over the last two weeks. Thus, these thoughts are assessed as suicidal or self-harmful thoughts. Viner et al. studied 188 patients with MS. At baseline, $8.3 \%$ of them endorsed ST, but this value increased to $22.1 \%$ over the course of six months [24]. Turner et al. also applied the Patient Health Questionnaire to 445 MS patients from the Veteran's Health Administration and obtained a surprisingly high prevalence of suicidal ideas (29.4\%) and persistent ST (35\%) [25]. In a larger group (3823 patients), Dickstein et al. found a suicidal behavior prevalence of $15 \%$ [26].

In another study conducted by Feinstein, 140 MS subjects were questioned using the Beck Suicide Scale and the Structured Clinical Interview for DSM IV Axis 1 Disorders, and $28.6 \%$ of participants had lifetime SI [7]. A German study concerning 867 people revealed that $22.1 \%$ of them answered "often," "very often," or "all the time" when asked "How often do you think about ending your life?" [27].

Lee et al. conducted a study to evaluate the prevalence of suicidal ideation in the general population. There were included 2054 people in the study, who were interviewed by telephone and $2.84 \%$ reported suicidal ideation in the last week [28].

The prevalence of SC in our group of patients was $10.03 \%$, the value that is the most consistent with the published data (11.76\%) as revealed in Ben-Zacharia's research who also used BDI-II [29]. So, it seems like 10 in 100 patients with MS would consider taking their lives even though they have not been previously diagnosed with any mental or other chronic illness that could increase their suicide risk.

In their review from ten years ago, Nock et al. described suicide and suicidal behavior in the United States and cross-nationally and showed that, in the general population, the rate of suicide is higher in males than in females. This pattern, despite the broad fluctuation, is characteristic for both the United States and other countries. Men die from suicide at a ratio ranging from $3: 1$ to $7.5: 1$. On the other hand, when it comes to suicidal behavior, the study revealed opposing results because the rate of nonfatal self-injury was more common in females [10]. Starting from the premise that men are more predisposed to complete suicide while women more often attempt suicide, we could expect that this behavior could be maintained in the MS population because of the progressive nature of the disease and the idea of being diagnosed with an incurable condition. However, this has not been proven as a rule. Some studies have shown that males with MS were more likely to have a suicidal ideation (odds ratio 1.52, $p<0.001$ ) than females [26], while others revealed high frequencies in both men and women for attempting suicide and suicide risk as SMR with no statistical significance $[13,23]$.

Fredrikson et al. revealed though a higher suicide risk in the first year of MS diagnosis and among younger males. $\mathrm{He}$ also found a suicide rate of 71 per every 100,000 persons/year among MS patients with a statistically significant difference between men and women $(p<0.001)$ [13].

Bronnum-Hansen et al. studied suicide among Danes with MS based on the Danish Multiple Sclerosis Registry updating a previous study concerning the same subject from 1992. They wanted to establish if the suicide risk among MS patients has changed since the mid-1900s. The SMR was overall similar for men and women (2.16 versus 2.07$)$. After 15 years from diagnosis, the excess suicide rate has changed only for women from 4.03 to 1.73 in the subsequent periods, while it remained nearly steady for men. The risk appears to have decreased in the 15 to 20 years after the diagnosis and 


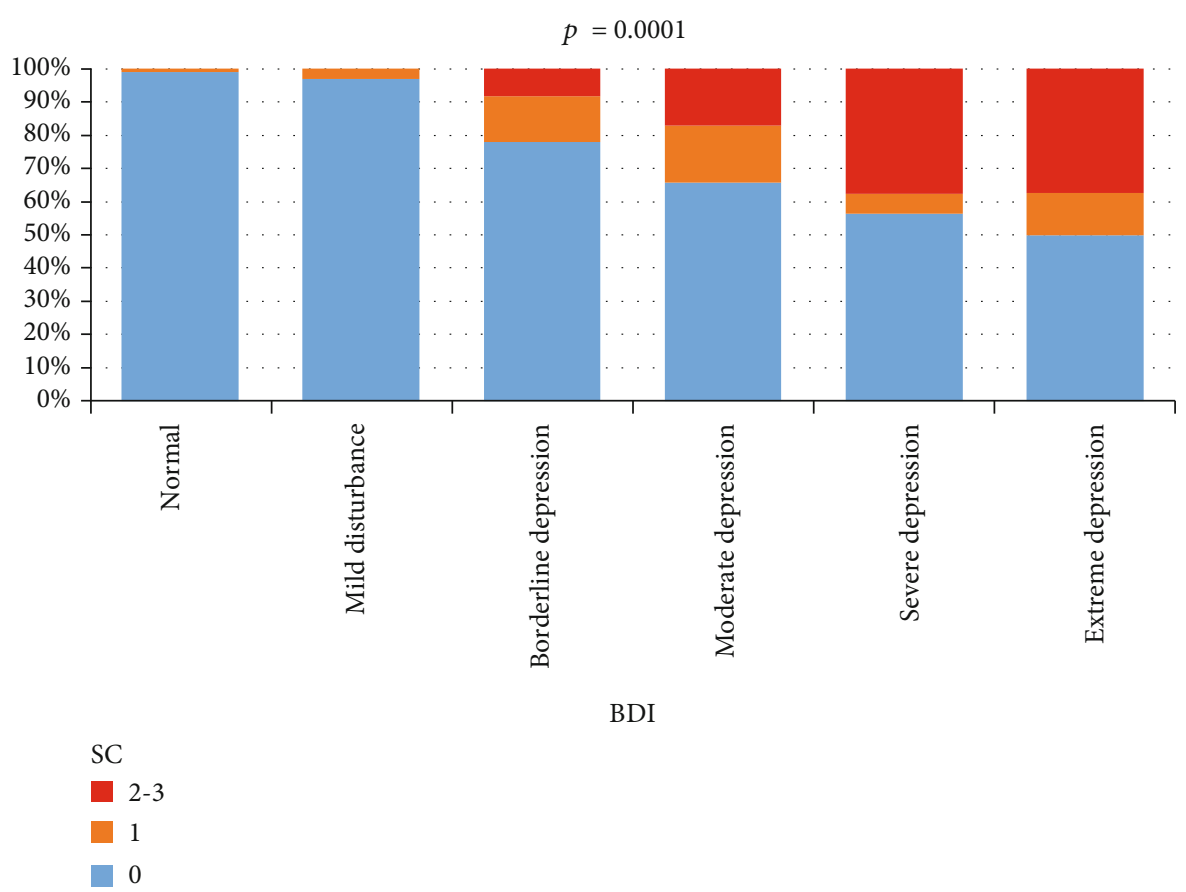

FIgURE 6: The relationship between SC and BDI score. Normal: <10 points, mild disturbance: 11-16 points, borderline depression: 17-20 points, moderate depression: 21-30 points, severe depression: $31-40$ points, and extreme depression: $>40$ points.

increased again after 20 years with a slightly higher risk for women (2.04 compared to 1.74). However, the calculated SMR had no statistically significant difference when comparing the two sexes or the variations over a time lapse in the same sex [21].

Our study included 112 men and 237 women. ST were found in $3.8 \%$ of women and $7.1 \%$ men, while SI have an opposite distribution with $5.9 \%$ in women and only $2.7 \%$ in men. However, no statistically significant differences between the two sexes regarding SI and ST were observed. Our results are consistent with those from the study performed by Turner and his colleagues who also did not return any statistical significance in a bivariate relation between suicidal ideas and gender [25].

Being diagnosed with a chronic condition such as MS is a burden, aside from the disabling character of the disease itself. Suicide in these patients could be seen as "being defeated by the long hard struggle to stay alive," as Brampton said in his book [30].

The World Health Organization defines disability as an "umbrella term" for a complex phenomenon that reflects the interactions between one's body functions and features of the society in which the person lives. It covers impairment (a problem in body function or structure), activity limitations (a difficulty in executing a task or action), and participation restrictions (a problem experienced when an individual becomes involved in life situations) [31].

Literature reports indicate that different types of disability such as limited activity, perceived and actual disabilities, and physical limitations are associated with suicidal ideation $[32,33]$.
Disability in MS is caused by the accumulation of different neurological impairments such as motor, sensory, coordination, gait, and visual symptoms as the disease concurrently affects distinct parts of the white and grey matter in the central nervous system. Aside from these manifestations, there is also a series of "hidden disabilities" that include difficulties with cognition, memory, mood, affect, pain, fatigue, sleep, bowel, bladder, and sexual functions that could be easily missed in a routine consultation [34]. Quantifying the disabilities in MS helps us to assess the disease progression, monitor clinical outcomes in individual patients for better care, and also evaluate the treatments' effects. The most universally used tool for measuring the disability and progression in MS is the EDSS developed by Kurtzke in 1983 [15].

After searching the literature for a direct relationship between SI and disability, more studies seem to lean towards a positive correlation. However, a large-scale study performed by Feinstein did not find a significant association [7].

Gaskill et al. performed a qualitative study in which they found that frustrations related to limited functioning and perceived loss of control were among the eight key themes identified in people with MS experiencing suicidal ideas, the last mentioned being the most common one [35].

Two more studies described that communication and swallowing difficulties and self-reported bowel and bladder problems were risk factors for SI [24, 25].

Other studies reported that moderate and severe levels of disability are risk factors for completed suicide in MS [36, 37].

A few studies revealed that the progressive MS types appear to be associated with higher levels of suicidal ideas 
because of the increased level of disability which characterize them, when compared with RRMS form [25, 38-40].

Regarding the disease duration, Bronnum-Hansen et al. found that SMR was higher for persons diagnosed for $>20$ years [21].

We also used the EDSS for our study group, and it was based on the neurological examination performed by the same doctor for all the study patients. We obtained a positive correlation between the presence and the severity of SC with the EDSS. Statistically significant values were found also when we correlated SC with PFS and with CFS, respectively. This draws the attention towards the fact that for our patients, losing control of the motor functions and not being able to coordinate one's movements are the most disabling situations that can lead to suicidal behavior. Although we did not find a positive correlation regarding the autonomic functions on EDSS and SC, it is important that almost 95\% of the patients with a bowel and bladder functional system of 0 had no SI or ST.

Relapses also add neurological impairments to the baseline of the functional status, even for a limited period of time, and we were not surprised that we found positive correlations between the number of relapses and SC. Also, the disease duration correlated positively with SI and ST which was consistent with the results of Bronnum-Hansen stated above [21].

Although the clinical forms of MS did not correlate with SC, it is worth mentioning that $100 \%$ of CIS patients had no SC, and more patients with SPMS (8.5\%) had SI compared to those with RRMS (4.1\%), the last finding being similar with the literature [25, 38-40].

Two studies conducted by Cerqueira and Brenner found correlations regarding the impact of education on the SC which agree partially with our results. Cerqueira et al. compared two groups of MS patients: one at risk of suicide and one without it (the control group) and they found that for the control group the education level was higher. Brenner et al. observed that the risk for attempting suicide was lower for both MS and non-MS patients with higher education levels, but the association for completed suicide was seen only in the non-MS group [23, 41].

On this matter, we found that $92.3 \%$ of patients with a university degree and $95.7 \%$ of the postgraduates had no ST, and SI were observed only in $1.9 \%$ of those with a university education, in contrast to $30.8 \%$ of patients with secondary education. This difference was statistically significant, so it seems that the lower the education level, the higher the incidence of SI. This could mean that a higher education level may give those patients a different perspective over the idea of living with MS. They could be more easily approached about the importance of treatment, the clinical and imaging follow-ups, and the possibilities of dealing with their disability. They may also have good coping mechanisms that help them gain confidence over the fact that life does not end with this diagnosis.

Of the DMT's used in our study, IFN beta raise the question whether there is a link between this therapy and suicide. In the literature, the relationship between IFN beta therapy and suicidal behavior is unclear. The first
IFN beta-1b trial concerning RRMS patients raised the suspicion as to whether this treatment could be linked to suicide because completed suicide and suicide attempt were documented for the treatment arms but not for the placebo group [42]. Additional studies have documented the association between IFN beta and the presence of symptoms for severe depression [43], and Goeb and his colleagues concluded in their review of IFN beta-1a and beta-1b that treatment itself has no greater risk of suicidal behavior [44]. However, in clinical practice, all patients undergoing treatment should be closely monitored for signs of suicidal thinking [18].

We did not see an influence of the DMT type on the prevalence of SC, but we found that $70.6 \%$ of the patients with ST and $66.7 \%$ of those with SI were treated with different types of IFN beta.

Social support is an important component in the psychological balance of each individual and even more so for a person suffering from various medical conditions. Lower social support levels are associated with an increased in vulnerability to stressors and a tendency towards physical and psychological health problems. Furthermore, people with suicidal behavior described less support from their friends and family $[45,46]$.

Arciniegas and Anderson stipulated that social isolation represents an increased risk factor for suicide in patients with neurological illnesses [47].

This finding was also reported in the case of MS. In the studies performed by Feinstein and Bronnum-Hansen and his colleagues, it was found that social isolation can be among the predictors for suicidal ideation in MS patients [7, 21].

In the study performed by Turner and his collaborators, lower levels of perceived social support were associated with suicidal ideas. He also found for the first time that lower income is a variable of the same equation. However, no relationship between living alone and suicidal behavior was noticed [25].

Long and Miller suggested that suicidal tendencies in MS patients could be predicted using the concept of family support [48].

Social support from family, friends, and significant others was found to be negatively correlated with SI in MS patients in a relatively recent study by Ariapooran and his team [49].

In another study conducted by Cerqueira et al., it was found that with respect to marital status, the risk of suicide was higher for single, separated, or widowed individuals when compared with the control group [41].

Although in our study group, occupational or marital status did not significantly influence the presence of SC, it is important to notice that of the 18 patients who express SI, $77 \%$ were married/living with a partner, $16.6 \%$ were unmarried, and 5.5\% were divorced. It seems that for the Romanian population having a diagnosis of a chronic disease like MS carries the biggest chance of developing SI when a person is married or living with a partner. This does not mean that our patients have little or no family support, but from their points of view, they may see themselves as a burden for their relatives. However, this should not lead us to draw any hasty conclusions and the problem should be studied within an 
extended framework of what social support means, not just the one offered by the family environment.

It is well known that mental illnesses carry a great risk for patients for harming or killing themselves and depression in all stages is no exception. The risk of suicidal ideas and attempts for patients suffering from mood disorders was estimated at $51 \%$ and $44 \%$, respectively, and $60 \%$ of completed suicide cases had a history of depressive episodes [50, 51].

Among all mental health conditions, depression is the most common one in the general population but is also an important and frequent comorbidity in other chronic pathologies such as neurodegenerative diseases and MS [12, 52, 53].

In the general population, the prevalence of depression varies from $20.6 \%$ in South America to $7.3 \%$ in Australia [52], but when it comes to MS, the prevalence is two- to threefold higher, ranging from $40 \%$ to $60 \%$ making depression the most frequent psychiatric manifestation of MS. In addition to this finding, people with MS suffer from more severe depressive symptoms hence the frequent association between depression, suicide, and MS [54-56].

The symptoms of depression in MS occur secondary to an interaction between the basic psychological structure of an individual and his reaction to the diagnosis, together with the changes following neurodegeneration, possible side effects of the medication, and the social support levels [43, 57-59].

The suicidal ideation is, in fact, a core symptom for major depression. In other words, referring to depression in MS, we could state that it is one of the strongest risk factors for suicidal ideation $[60,61]$

This association between depression and suicide was found by Cerqueira et al. in their study in which they also observed the presence of other psychiatric disorders such as bipolar disorder, psychotic syndrome, abuse and dependence on drugs, and bulimia nervosa in the suicide risk group [41].

Other researchers such as Viner, Turner, Fisk, and their colleagues endorsed the same correlation [24, 25, 62]. Fisk et al. studied the prevalence of depression, bipolar disorder, and attempted suicide among patients who used hospital psychiatric resources and compared those characteristics between the MS and non-MS groups. They concluded that bipolar disorders and depression on one hand and the frequency of suicide attempts on the other were two and, respectively, three times greater in the MS patients group [62]. Turner et al. found that suicidal ideas were significantly more common among patients with a higher depression severity score [25].

It has been found that depression not only is a risk factor itself for suicidal ideation but also acts as a mediator among other determinants of suicidal behavior. In their study, Lewis and his colleagues observed that depressive symptoms had an indirect effect on the relationship between actual and perceived disability and suicide ideation in MS [33].

In our study, we found the strongest correlation between the presence and severity of SC and the total score of the BDI-II questionnaire $(p=0.0001)$. No SC were found for $98.9 \%$ of those with a score $<10$. SI were present in $37.5 \%$ of those with severe depression, while those suffering from mild disturbances or those without any mood disorders had no SI. These results are consistent with preexisting studies mentioned before and perpetuate the idea of suicide being the most dramatic facet of major depression.

To improve a further research, we could extend our statistics not only for suicidal ideation but also for suicide attempts and completed suicide at a national level, apply more tests or questionnaires concerning suicidal behavior, searching for persistent suicidal ideation over a longer time period, and after treating the subsequent depressive symptoms. We could also study if any other concomitant psychiatric disease influences the suicidal behavior or if MS has an effect on a preexisting psychiatric pathology regarding the suicidal behavior.

\section{Conclusions}

The prevalence of SC is higher in patients with MS compared to the general population. The main determinants for SC that we found are depression, an increased level of disability, a high number of recurrences, a longer duration of illness, and a low level of education.

Suicide is a tragedy, but we have the tools to prevent it. Searching for the determinants of suicidal ideation should now be a part of the routine examination. Among all of them, depression is the most common and highly associated with SC. A big step forward in avoiding suicide among MS patients is to suspect and diagnose depression in an early stage because, unlike other facets of MS, it is the most receptive to treatment.

\section{Abbreviations}

BDI-II: Beck depression inventory-II

CFS: Cerebellar functional score

CIS: Clinically isolated syndrome

DMT: Disease-modifying therapy

EDSS: Expanded disability status scale

IFN: Interferon

MS: $\quad$ Multiple sclerosis

PFS: Pyramidal functional score

RRMS: Relapsing-remitting multiple sclerosis

SC: $\quad$ Suicidal concerns

SI: $\quad$ Suicidal intentions

SMR: Standardized mortality ratio

SPMS: Secondary progressive multiple sclerosis

ST: $\quad$ Suicidal thoughts.

\section{Data Availability}

The data that support the findings of this study are available from the corresponding author, N.Ş., upon reasonable request.

\section{Ethical Approval}

The authors assert that all procedures contributing to this work comply with the ethical standards of the relevant national and institutional committees on human 
experimentation and with the Helsinki Declaration of 1975, as revised in 2008.

\section{Conflicts of Interest}

The authors declare that they have no conflicts of interest.

\section{Authors' Contributions}

The first two authors contributed equally to the manuscript and both should be viewed as first author. A.R. analyzed/interpreted the data and drafted the manuscript. R.B. designed the study and critically revised the manuscript. N.Ş. collected and analyzed the data. S.A. collected the data. Z.B. revised the manuscript. L.B. revised the manuscript for English language. S.V. analyzed the data with the statistical program. S.M. has contributed to the study design, data collection, data interpretation, and critical revision of the manuscript. A.M. collected the data and critical revision of the manuscript. All authors met the following authorship criteria: (1) substantial contribution to the conception and design of or acquisition of data or analysis and interpretation of data, (2) drafting the article or revising it critically for important intellectual content, and (3) final approval of the version to be published.

\section{References}

[1] S. Maier, M. Buruian, A. Maier et al., "The determinants of depression in a Romanian cohort of multiple sclerosis patients," Acta Neurologica Belgica, vol. 116, no. 2, pp. 135$143,2016$.

[2] J. M. Charcot, Lectures on the Disease of the Nervous System, Henry C Lea, Philadelphia, 1879.

[3] C. Diaz-Olavarrieta, J. L. Cummings, J. Velazquez, and C. Garcia de la Cadena, "Neuropsychiatric manifestations of multiple sclerosis," The Journal of Neuropsychiatry and Clinical Neurosciences, vol. 11, no. 1, pp. 51-57, 1999.

[4] O. Ghaffar and A. Feinstein, "The neuropsychiatry of multiple sclerosis: a review of recent developments," Current Opinion in Psychiatry, vol. 20, no. 3, pp. 278-285, 2007.

[5] L. C. Politte, J. C. Huffman, and T. A. Stern, "Neuropsychiatric manifestations of multiple sclerosis," The Primary Care Companion to The Journal of Clinical Psychiatry, vol. 10, no. 4, pp. 318-324, 2008.

[6] L. A. Rolak, "Multiple sclerosis: it's not the disease you thought it was," Clinical Medicine \& Research, vol. 1, no. 1, pp. 57-60, 2003.

[7] A. Feinstein, "An examination of suicidal intent in patients with multiple sclerosis," Neurology, vol. 59, no. 5, pp. 674678, 2002.

[8] A. Feinstein, "Multiple sclerosis and depression," Multiple Sclerosis, vol. 17, no. 11, pp. 1276-1281, 2011.

[9] Z. Samaan, M. Bawor, B. B. Dennis et al., "Exploring the determinants of suicidal behavior: conventional and emergent risk (DISCOVER): a feasibility study," Pilot and Feasibility Studies, vol. 1, p. 17, 2015.

[10] M. K. Nock, G. Borges, E. J. Bromet, C. B. Cha, R. C. Kessler, and S. Lee, "Suicide and suicidal behavior," Epidemiologic Reviews, vol. 30, no. 1, pp. 133-154, 2008.
[11] World Health Organization, WHO Suicide Data, World Health Organization, Geneva, 2015.

[12] S. S. Coughlin and L. Sher, "Suicidal behavior and neurological illnesses," Journal of Depression and Anxiety, vol. 2, no. 1, article 12443, 2013.

[13] S. Fredrikson, Q. Cheng, G. X. Jiang, and D. Wasserman, "Elevated suicide risk among patients with multiple sclerosis in Sweden," Neuroepidemiology, vol. 22, no. 2, pp. 146-152, 2003.

[14] C. H. Polman, S. C. Reingold, B. Banwell et al., "Diagnostic criteria for multiple sclerosis: 2010 revisions to the McDonald criteria," Annals of Neurology, vol. 69, no. 2, pp. 292-302, 2011.

[15] J. F. Kurtzke, "Rating neurologic impairment in multiple sclerosis: an expanded disability status scale (EDSS)," Neurology, vol. 33, no. 11, pp. 1444-1452, 1983.

[16] A. T. Beck, C. H. Ward, M. Mendelson, J. Mock, and J. Erbaugh, "An inventory for measuring depression," Archives of General Psychiatry, vol. 4, no. 6, pp. 561-571, 1961.

[17] Statistical Package for Social Sciences [Computer Program]. Version 22, IBM Corp, Armonk, NY, 2013.

[18] A. Feinstein and B. Pavisian, "Multiple sclerosis and suicide," Multiple Sclerosis, vol. 23, no. 7, pp. 923-927, 2017.

[19] N. Koch-Henriksen, H. Bronnum-Hansen, and E. Stenager, "Underlying cause of death in Danish patients with multiple sclerosis: results from the Danish Multiple Sclerosis Registry," Journal of Neurology, Neurosurgery, and Psychiatry, vol. 65, no. 1, pp. 56-59, 1998.

[20] E. N. Stenager, E. Stenager, N. Koch-Henriksen et al., "Suicide and multiple sclerosis: an epidemiological investigation," Journal of Neurology, Neurosurgery, and Psychiatry, vol. 55, no. 7, pp. 542-545, 1992.

[21] H. Brønnum-Hansen, E. Stenager, E. N. Stenager, and N. Koch-Henriksen, "Suicide among Danes with multiple sclerosis," Journal of Neurology, Neurosurgery, and Psychiatry, vol. 76, no. 10, pp. 1457-1459, 2005.

[22] M. L. Sumelahti, M. Hakama, I. Elovaara, and E. Pukkala, "Causes of death among patients with multiple sclerosis," Multiple Sclerosis, vol. 16, no. 12, pp. 1437-1442, 2010.

[23] P. Brenner, S. Burkill, J. Jokinen, J. Hillert, S. Bahmanyar, and S. Montgomery, "Multiple sclerosis and risk of attempted and completed suicide-a cohort study," European Journal of Neurology, vol. 23, no. 8, pp. 1329-1336, 2016.

[24] R. Viner, S. B. Patten, S. Berzins, A. G. Bulloch, and K. M. Fiest, "Prevalence and risk factors for suicidal ideation in a multiple sclerosis population," Journal of Psychosomatic Research, vol. 76, no. 4, pp. 312-316, 2014.

[25] A. P. Turner, R. M. Williams, J. D. Bowen, D. R. Kivlahan, and J. K. Haselkorn, "Suicidal ideation in multiple sclerosis," Archives of Physical Medicine and Rehabilitation, vol. 87, no. 8, pp. 1073-1078, 2006.

[26] L. Dickstein, A. Nowacki, and A. Viguera, "Prevalence and predictors of suicidal ideation in epilepsy and multiple sclerosis (P 7.040)," Neurology, vol. 82, no. 10, 2014.

[27] J. Strupp, C. Ehmann, M. Galushko et al., "Risk factors for suicidal ideation in patients feeling severely affected by multiple sclerosis," Journal of Palliative Medicine, vol. 19, no. 5, pp. 523-528, 2016.

[28] J. I. Lee, M. B. Lee, S. C. Liao et al., "Prevalence of suicidal ideation and associated risk factors in the general population," Journal of the Formosan Medical Association, vol. 109, no. 2, pp. $138-147,2010$. 
[29] A. Ben-Zacharia, "Suicide ideation in multiple sclerosis (P2.191)," Neurology, vol. 86, no. 16, 2016.

[30] S. Brampton, Shoot the Damn Dog: A Memoir of Depression, W.W. Norton\& Company, New York, NY, USA, 2008.

[31] World Health Organisation, Disabilities, 2018, http://www. who.int/topics/disabilities/en/.

[32] Y. Chen Lin, C. Yeh Chen, M. Shyan Lin, P. Han Chen, and M. Yen Chen, "Prevalence of suicidal ideation and associated risk factors among adults with disabilities," Neuropsychiatry, vol. 7, no. 4, pp. 406-415, 2018.

[33] V. M. Lewis, K. Williams, C. Koko, J. Woolmore, C. Jones, and T. Powell, "Disability, depression and suicide ideation in people with multiple sclerosis," Journal of Affective Disorders, vol. 208, pp. 662-669, 2017.

[34] G. Giovannoni, J. F. Foley, and D. W. Brandes, "Hidden disabilities in multiple sclerosis-the impact of multiple sclerosis on patients and their caregivers," European Neurological Review, vol. 8, pp. 2-9, 2013.

[35] A. Gaskill, F. W. Foley, J. Kolzet, and M. A. Picone, "Suicidal thinking in multiple sclerosis," Disability and Rehabilitation, vol. 33, no. 17-18, pp. 1528-1536, 2011.

[36] E. N. Stenager, N. Koch-Henriksen, and E. Stenager, "Risk factors for suicide in multiple sclerosis," Psychotherapy and Psychosomatics, vol. 65, no. 2, pp. 86-90, 1996.

[37] A. L. Berman and L. Samuel, "Suicide among people with multiple sclerosis," Neurorehabilitation and Neural Repair, vol. 7, no. 2, pp. 53-62, 1993.

[38] L. Chwastiak, D. M. Ehde, L. E. Gibbons, M. Sullivan, J. D. Bowen, and G. H. Kraft, "Depressive symptoms and severity of illness in multiple sclerosis: epidemiologic study of a large community sample," The American Journal of Psychiatry, vol. 159, no. 11, pp. 1862-1868, 2002.

[39] M. Dennis, S. Baillon, T. Brugha, J. Lindesay, R. Stewart, and H. Meltzer, "The influence of limitation in activity of daily living and physical health on suicidal ideation: results from a population survey of Great Britain," Social Psychiatry and Psychiatric Epidemiology, vol. 44, no. 8, pp. 608-613, 2009.

[40] G. Sarısoy, M. Terzi, K. Gümüş, and O. Pazvantoğlu, "Psychiatric symptoms in patients with multiple sclerosis," General Hospital Psychiatry, vol. 35, no. 2, pp. 134-140, 2013.

[41] A. C. de Cerqueira, P. S. Andrade, J. M. Godoy-Barreiros, A. C. de Oliveira e Silva, and A. E. Nardi, "Risk factors for suicide in multiple sclerosis: a case-control study," Jornal Brasileiro de Psiquiatria, vol. 64, no. 4, pp. 303-306, 2015.

[42] The IFNB Multiple Sclerosis Study Group and the University of British Columbia MS/MRI Analysis Group, "Interferon beta-lb in the treatment of multiple sclerosis: final outcome of the randomized controlled trial," Neurology, vol. 45, no. 7, pp. 1277-1285, 1995.

[43] Y. D. Fragoso, E. R. Frota, J. S. Lopes et al., "Severe depression, suicide attempts, and ideation during the use of interferon beta by patients with multiple sclerosis," Clinical Neuropharmacology, vol. 33, no. 6, pp. 312-316, 2010.

[44] J. L. Goeb, C. Even, G. Nicolas, B. Gohier, F. Dubas, and J. B. Garré, "Psychiatric side effects of interferon-beta in multiple sclerosis," European Psychiatry, vol. 21, no. 3, pp. 186-193, 2006.

[45] J. A. Boscarino, "Post-traumatic stress and associated disorders among Vietnam veterans: the significance of combat exposure and social support," Journal of Traumatic Stress, vol. 8, no. 2, pp. 317-336, 1995.
[46] G. Endo, H. Tachikawa, Y. Fukuoka et al., "How perceived social support relates to suicidal ideation: a Japanese social resident survey," The International Journal of Social Psychiatry, vol. 60, no. 3, pp. 290-298, 2014.

[47] D. B. Arciniegas and C. A. Anderson, "Suicide in neurologic illness," Current Treatment Options in Neurology, vol. 4, no. 6, pp. 457-468, 2002.

[48] D. D. Long and B. Miller, "Suicidal tendency and multiple sclerosis," Health \& Social Work, vol. 16, no. 2, pp. 104-109, 1991.

[49] S. Ariapooran, M. Rajabi, and A. Goodarzi, "Relationship between social support, time perspective and suicide ideations in patients with multiple sclerosis," Asian Social Science, vol. 12, no. 8, pp. 192-200, 2016.

[50] M. K. Nock, I. Hwang, N. A. Sampson, and R. C. Kessler, "Mental disorders, comorbidity and suicidal behavior: results from the National Comorbidity Survey Replication," Molecular Psychiatry, vol. 15, no. 8, pp. 868-876, 2010.

[51] P. Courtet and J. Lopez-Castroman, "Antidepressants and suicide risk in depression," World Psychiatry, vol. 16, no. 3, pp. 317-318, 2017.

[52] G. Y. Lim, W. W. Tam, Y. Lu, C. S. Ho, M. W. Zhang, and R. C. Ho, "Prevalence of depression in the community from 30 countries between 1994 and 2014," Scientific Reports, vol. 8, no. 1, p. 2861, 2018

[53] S. Maier, R. Balasa, M. Buruian, A. Maier, and Z. Bajko, "Depression in multiple sclerosis-review," Romanian Journal of Neurology, vol. 14, no. 1, pp. 22-29, 2015.

[54] A. D. Sadovnick, R. A. Remick, J. Allen et al., "Depression and multiple sclerosis," Neurology, vol. 46, no. 3, pp. 628-632, 1996.

[55] A. Solari, A. Motta, L. Mendozzi et al., "Italian version of the Chicago multiscale depression inventory: translation, adaptation and testing in people with multiple sclerosis," Neurological Sciences, vol. 24, no. 6, pp. 375-383, 2004.

[56] M. T. Wallin, J. A. Wilken, A. P. Turner, R. M. Williams, and R. Kane, "Depression and multiple sclerosis: review of a lethal combination," Journal of Rehabilitation Research and Development, vol. 43, no. 1, pp. 45-62, 2006.

[57] M. A. Raskind, "Diagnosis and treatment of depression comorbid with neurologic disorders," The American Journal of Medicine, vol. 121, no. 11, pp. S28-S37, 2008.

[58] S. B. Patten, G. Francis, L. M. Metz, M. Lopez-Bresnahan, P. Chang, and F. Curtin, "The relationship between depression and interferon beta-1a therapy in patients with multiple sclerosis," Multiple Sclerosis, vol. 11, no. 2, pp. 175-181, 2005.

[59] A. Feinstein, The Clinical Neuropsychiatry of Multiple Sclerosis, Cambridge University Press, New York, NY, USA, 2007.

[60] M. Pompili, A. Forte, M. Palermo et al., "Suicide risk in multiple sclerosis: a systematic review of current literature," Journal of Psychosomatic Research, vol. 73, no. 6, pp. 411-417, 2012.

[61] R. A. Marrie, "What is the risk of suicide in multiple sclerosis?," Multiple Sclerosis, vol. 23, no. 6, pp. 755-756, 2017.

[62] J. D. Fisk, S. A. Morehouse, M. G. Brown, C. Skedgel, and T. Jock Murray, "Hospital-based psychiatric service utilization and morbidity in multiple sclerosis," The Canadian Journal of Neurological Sciences, vol. 25, no. 3, pp. 230-235, 1998. 


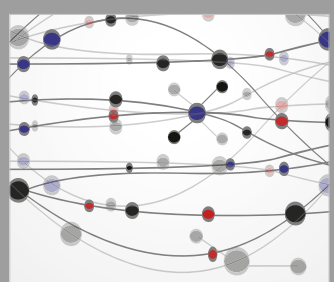

The Scientific World Journal
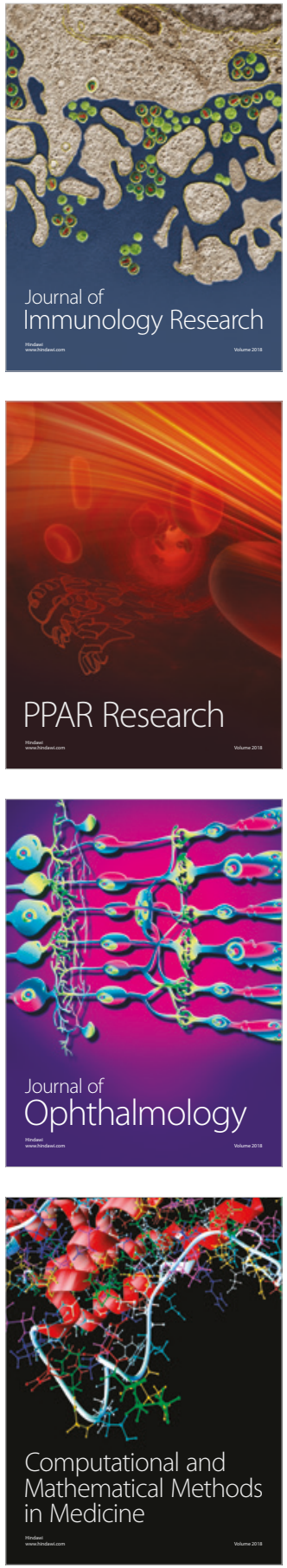

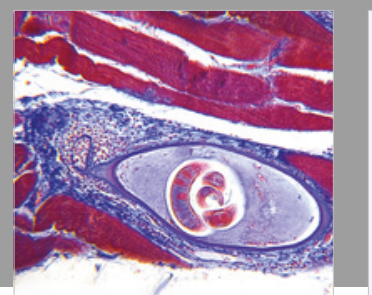

Gastroenterology Research and Practice

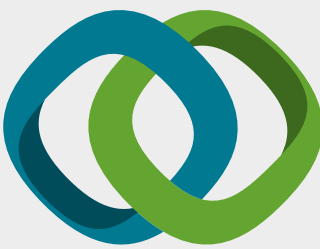

\section{Hindawi}

Submit your manuscripts at

www.hindawi.com
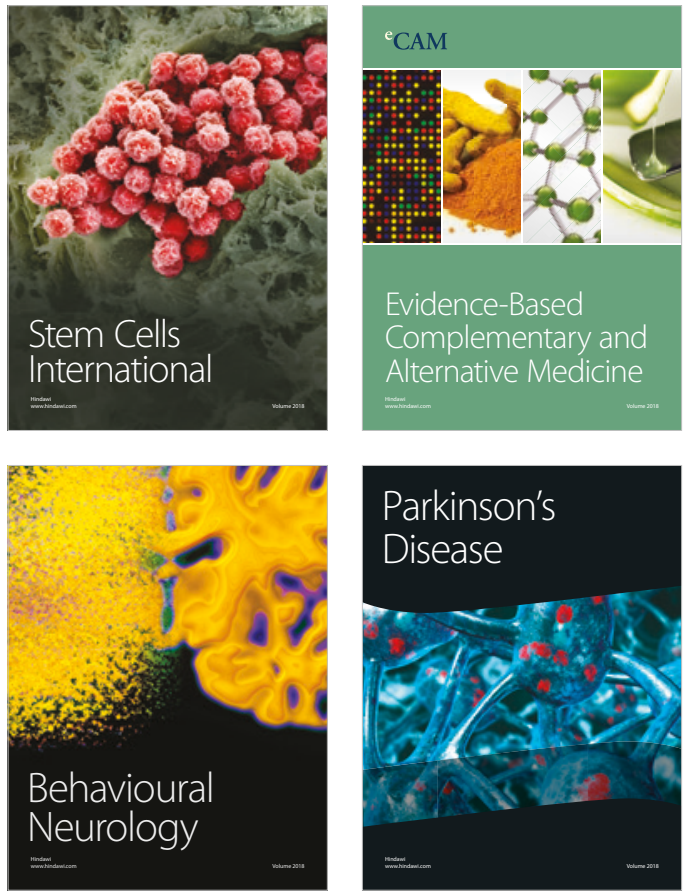

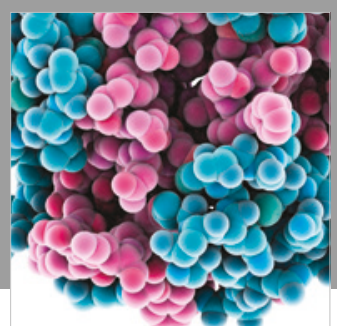

ournal of

Diabetes Research

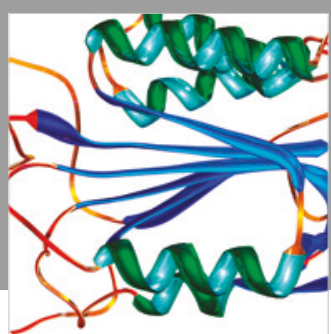

Disease Markers
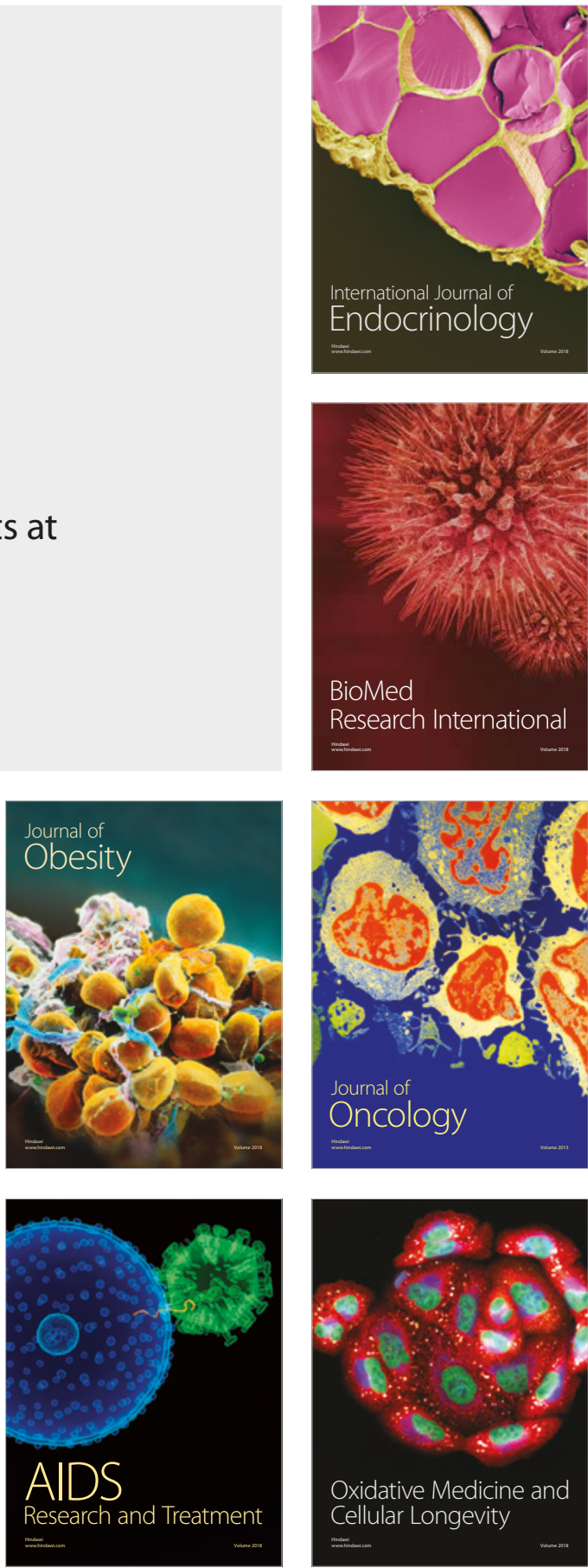